J3eA - Vol. 2 - 13 (2003).

DOI : 10.1051/bib-j3ea:2003013

\title{
LE DEUG STPI à Caen : initialisation d'innovations pédagogiques
}

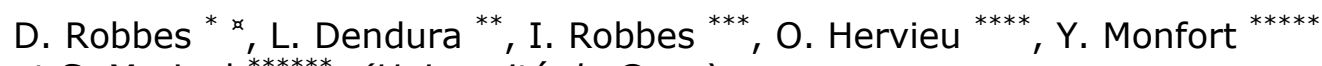 \\ et S. Marinel ${ }^{* * * * * *}$ (Université de Caen) \\ Mis en ligne le 23 septembre 2003.
}

\begin{abstract}
Résumé
Le DEUG Sciences et Technologies pour l'Ingénieur (STPI) de l'Université de Caen a accueilli sa première promotion d'étudiants à la rentrée 2000. L'objet de cet article est de décrire quelques innovations pédagogiques proposées dans le document d'habilitation du diplôme et leur première mise en place. Les innovations portent sur des travaux pratiques, le travail sur le projet professionnel et l'introduction d'une unité d'histoire des sciences et des techniques.

Mots-clés : projet professionnel, histoire des sciences et des techniques, auto-évaluation.

(C) EDP Sciences, 2003.
\end{abstract}

Niveau de connaissances requis. Baccalauréat scientifique.

Niveau des étudiants. Première année de DEUG Sciences et Technologie.

* Didier Robbes ${ }^{1}$ est professeur d'électronique à l'Université de Caen. Il est Directeur des études du DEUG STPI caennais créé à la rentrée 2000. Il effectue sa recherche au GREYC UMR 6072.

a e-mail : Didier.Robbes@greyc.ismra.fr (auteur de correspondance)

** Laurence Dendura ${ }^{1}$ est conseillière d'orientation psychologique au Centre d'Information et d'Orientation de Caen II.

*** Isabelle Robbes ${ }^{1}$ est conseillière d'orientation psychologique au Centre d'Information et d'Orientation de Saint-Lô.

**** Olivier Hervieu ${ }^{1}$ a été étudiant en DEUG STPI, promotion 2000.Il a obtenu la licence EEA de l'université de Caen en juin 2003.

***** Yves Montfort ${ }^{1}$ est maître de conférences hors classe à l'Université de Caen dans les disciplines de l'électronique, effectuant sa recherche au GREYC UMR 6072.

****** Sylvain Marinel ${ }^{1}$ est maître de conférences à l'Université de Caen en sciences des matériaux, effectuant sa recherche au CRISMAT UMR 6508.

\footnotetext{
${ }^{1}$ Université de Caen, Campus Côte de Nacre, bd Maréchal Juin, F-14050 Caen, France.

Article available at http://www.j3ea.org or http://dx.doi.org/10.1051/bib-j3ea:2003013
} 


\section{Introduction}

Dans le contexte de la diminution des effectifs en sciences, et notamment en EEA (Électronique, Électrotechnique et Automatique), le département de Physique-EEA de l'Université de Caen a néanmoins souhaité compléter sa filière EEA par l'introduction d'un DEUG STPI. Nous mettons ici l'accent ce que nous estimons être des innovations pédagogiques ou pouvant s'apparenter à une démarche qualité [1].

L'article s'articule en deux grandes parties. La première porte sur des innovations hors des champs disciplinaires habituels, et différant sensiblement de la pratique commune : élément « harmonisation des origines et consolidation des connaissances » et élément « projet professionnel ». Cette première partie se termine par la description d'un procédé d'évaluation active en contrôle continu. En seconde partie, nous décrivons nos séquences plus classiques consacrées à l'EEA et le lien avec la chimie, par piles et accumulateur, puis notre approche de l'histoire des sciences et techniques. La conclusion inclura des réactions des élèves de la première promotion.

\section{Innovations hors disciplines}

\subsection{Harmonisation des origines et consolidation des connaissances}

Cette séquence, vise à exploiter la diversité d'origine des étudiants (bac S, STI-électronique, STIélectrotechnique, STL-physique, étudiants étrangers). Sa devise peut s'exprimer ainsi : « se connaître différences/complémentarités - pour s'enrichir ». Les documents de référence de cet élément d'enseignement (24 $\mathrm{h}$ TD) ne sont autres que les programmes officiels des classes de première et de terminale (math-physiquechimie). Les idées de base y sont les suivantes : après examen des divers programmes de leur cursus immédiatement antérieur au DEUG, les étudiants doivent dégager les points communs et les différences entre leurs formations secondaires. Ils doivent également noter les connaissances et méthodes exigibles clairement mentionnées dans le descriptif des programmes. Ce point est important, car il constitue à notre avis un lien important entre les formations/enseignants du secondaire et le supérieur. Chaque enseignant en premier cycle du supérieur doit ou devrait savoir se référer aux programmes en cours pour l'obtention du bac. En cas d'écart trop important et/ou d'oubli intempestif des connaissances de base par les étudiants, sans doute ceux-ci peuvent-ils être plus clairement placés face aux réalités et exigences des formations du supérieur, eu égard à ce qui est spécifié comme exigible d'un titulaire du baccalauréat [2] !

La forme prise pendant les deux heures hebdomadaires est la donnée de mini-cours (20 min) par les étudiants, avec notations sur grille de critères travaillée et définie au préalable par le groupe. Pour établir cette grille de critères, il est par exemple demandé aux étudiants de formuler ce qu'est pour eux un bon professeur ou un professeur médiocre. L'analyse des réponses permet de dégager assez rapidement un ensemble de critères cohérents. Le sujet des mini-cours est ciblé sur la différence des programmes (harmonisation) et sur les notions mathématiques particulièrement importantes (consolidation). À l'issue de chaque mini-cours, l'enseignant précise où, dans le cursus, la notion présentée sera reprise, approfondie, utilisée. Enfin, l'extraction d'une note pour chacun des étudiants est obtenue suivant un protocole particulier, faisant intervenir chaque auditeur du cours, qui, après chaque audition d'un mini-cours, doit remplir la grille de critères précédemment définie. Pour chaque ligne de critère, chaque auditeur doit choisir si l'étudiant en situation de professeur a très bien, bien, moyennement, peu, ou pas du tout satisfait au critère. À l'issue de toutes les auditions, chaque étudiant liste à nouveau ses grilles. L'enseignant récupère alors l'ensemble des listes. La note finale attribuée à chaque étudiant résulte alors pour $2 / 3$ de la moyenne du groupe et pour $1 / 3$ de l'enseignant. Notons que pour la première promotion d'étudiants ayant suivi cette séquence (18), l'écart moyen entre la note attribuée par l'enseignant et celle attribuée par le groupe était de -0,4 points, et l'écart maximal était de 1,5 points dans un sens ou dans l'autre.

\subsection{Projet professionnel}

Depuis quelques années, des expériences autour du travail du projet professionnel en DEUG ont été tentées dans plusieurs universités, notamment sous l'impulsion de Marie-Claire Mery à Orsay [3]. L'objectif essentiel du travail autour du projet professionnel dans le DEUG STPI de Caen est très voisin de celui affiché par l'université d'Orsay : «le projet professionnel a pour objectif d'aider les étudiants à devenir acteur de leur orientation et de 
leur avenir professionnel ». En revanche, notre approche est différente, résultant notamment de l'intégration de conseillers d'orientation psychologues pour assurer cette séquence, alors que ce travail est assuré par des collègues enseignants-chercheurs volontaires dans les autres établissements, avec une reconnaissance incertaine en terme de service statutaire et de compétences professionnelles dans le domaine de l'orientation. Les séquences, d'une durée totale de 12 heures, permettent de mener une réflexion approfondie de façon individuelle et collective sur les différentes composantes du projet personnel et professionnel : connaissance de soi, valeurs, intérêts, compétences personnelles ou professionnelles acquises. La synthèse de ces éléments permet de dégager ou de préciser un ou plusieurs projets professionnels cohérents. Chacun des étudiants peut, après avoir fait les recherches documentaires appropriées, définir un ou plusieurs parcours de formation selon ses objectifs. Il faut noter que ce projet peut même remettre en cause le choix initial de DEUG STPI si cela s'avère nécessaire, mais rappelons que cette démarche s'inscrit parfaitement dans l'esprit des textes Bayrou actuellement en vigueur. Enfin, et là nous retrouvons la méthode de nos collègues, il est demandé à chaque étudiant de réaliser et de présenter à ses pairs, un poster consécutif à ses recherches documentaires portant sur le métier ou le secteur d'activité professionnelle de son choix. L'évaluation des posters donne aux étudiants des points de jury, qui pourront, le cas échéant, leur être fort utiles.

\subsection{Auto-évaluation guidée}

À coté du protocole traditionnel des contrôles continus, nous avons introduit le protocole alternatif suivant : les étudiants passent une épreuve courte (durée $1 \mathrm{~h}$ ). À son issue, l'enseignant répartit les étudiants par groupe de cinq à six, leur confie un corrigé avec barème et cinq à six copies anonymes que chaque étudiant du groupe devra noter. L'enseignant en séance répond aux multiples interrogations et incertitudes des étudiants. La note finale résulte de la moyenne des notes, après validation par l'enseignant. Cette pratique n'est certes pas complètement originale, mais semble bien appréciée par les étudiants, probablement au travers de l'aspect de mise en situation de correcteur, assimilable à un processus de remédiation cognitive.

\section{Innovations dans le champ STPI}

\subsection{Travaux pratiques}

Les travaux pratiques d'EEA du premier semestre ont pour but de faire découvrir quelques fonctions de base, quelques grandeurs fondamentales dans ces trois disciplines. Ils sont axés sur l'observation, la mesure et l'analyse de signaux relevés sur des maquettes préalablement réalisées. Pour donner un fil conducteur à la série des travaux pratiques, on a choisi de créer les dispositifs qui assurent les fonctions principales que l'on rencontre dans une télécommande de modèles réduits.

Le premier travail pratique concerne une seule commande : la position d'un « manche », associé à un potentiomètre est traduite par une durée d'impulsion électrique, répétée à la cadence de $20 \mathrm{~ms}$.

Le second travail pratique fait constater l'espace de temps inutilisé entre les impulsions. Il introduit la notion de multiplexage temporel en intercalant dans cet intervalle de temps d'autres impulsions dont la durée est associée à de nouveaux «manches » de commande. Le démultiplexage qui suit introduit les fonctions et circuits logiques nécessaires à cette opération.

Le troisième travail pratique analyse le fonctionnement d'un servo-mécanisme asservi en position. On met en évidence les grandeurs de commande et de sortie ainsi que la grandeur d'erreur obtenue en comparant les précédentes, erreur qui permet de corriger la sortie et d'asservir la position.

Le quatrième travail pratique, utilisant deux moteurs couplés, permet de mesurer les caractéristiques essentielles des moteurs à courant continu. On y voit les grandeurs que sont les f.e.m. en fonction de la vitesse de rotation, le couple moteur, la résistance, la puissance électrique et la puissance mécanique, ainsi que le rendement.

Quatre séances de travaux pratiques ont été mises en place au premier semestre sur le thème du stockage et de la restitution de l'énergie électrique. La première séance consiste à effectuer une recherche bibliographique sur ce thème, la ressource principale étant les moteurs de recherche sur le réseau Internet ainsi que le catalogue informatisé de l'Université de Caen. Cette recherche est bien entendu balisée puisque les étudiants disposent d'un 
support écrit leur demandant, en particulier, de répondre à des questions bien précises (définitions de grandeurs fondamentales, telles que les potentiels standards, ou technologiques, comme la capacité massique d'un accumulateur). Les trois séances suivantes ont pour objet de mettre en pratique les notions abordées lors de la recherche bibliographique. Lors du deuxième TP, les étudiants déterminent expérimentalement les potentiels standards de différents métaux ( $\mathrm{Ag}, \mathrm{Al}, \mathrm{Cd}, \mathrm{Ni}, \mathrm{Pb}, \mathrm{Zn}, \mathrm{Mg}$ ). Ils classent ensuite ces différents métaux en fonction de leurs pouvoirs oxydants. Les étudiants fabriquent, lors du troisième TP, une pile Leclanché et mesurent la différence de potentiels à ses bornes. Le phénomène d'oxydo-réduction qu'est la corrosion du fer est étudié plus en détail lors du quatrième TP.

\subsection{Histoire des sciences et des techniques}

Il est souvent noté dans les colloques et rencontres traitant du désengouement des sciences par nos jeunes que l'histoire des sciences est le parent pauvre de la culture générale des bacheliers, y compris les bacheliers scientifiques. Nous avons donc proposé d'introduire une unité de culture générale et d'expression au deuxième semestre de notre DEUG portant sur l'histoire des sciences et des techniques. Nous avons procédé comme suit : un questionnaire initial autour de l'histoire des sciences et des techniques, deux séances de réflexion sur les diverses façons d'aborder le sujet, sélection de sujets d'exposés et audition de ceux-ci ( $1 / 2$ heure). Concernant notre questionnaire initial, nous ne le détaillons pas ici, les résultats portent sur un effectif trop réduit pour être statistiquement significatifs. Notons seulement qu'il dénote une représentation initiale assez pauvre de l'histoire des sciences et des techniques, comme en témoigne l'ignorance à $100 \%$ de la signification du mot « épistémologie ». En seconde partie de cet élément, nous avons fait dégager par le groupe d'étudiants en sciences quelques possibilités d'approche de cette discipline (chronologique, thématique, socio-économique...), de façon à faire percevoir l'ampleur de cette discipline, telle qu'il serait illusoire de prétendre couvrir l'ensemble du domaine en une vingtaine d'heures de cours magistraux. Enfin, puisqu'il était souhaitable d'intégrer une partie « expression » dans cette unité de culture générale et d'expression, nous avons finalement retenu une approche thématique se concrétisant par une série d'exposés. Pour effectuer leur présentation, les étudiants disposent d'un ensemble PC-vidéo projecteur (connexion à Internet également possible en séance). Ils viennent à leur séance munis de leur CD-ROM ou disquette. Notons que le procédé classique par transparents est également possible. Néanmoins, les intérêts du support informatique sont multiples, en particulier ils sont attractifs pour les étudiants et la rediffusion sur le réseau internet des exposés particulièrement réussis est envisageable. Concernant l'évaluation du travail de l'étudiant, le protocole est assez semblable à celui présenté au paragraphe 2-1 avec des critères spécifiques à l'histoire des sciences et des techniques.

\section{Conclusion}

En guise de conclusion, il nous a semblé intéressant de reporter une courte synthèse des réactions des étudiants, reformulée par l'un d'eux :

«La séquence de consolidation des origines nous a permis de revoir dans un premier temps les enseignements dispensés au cours de notre cursus pré-bac. Dans un deuxième temps l'élargissement vers les domaines où ces enseignements seront utilisés permet d'avoir une idée plus précise de la réalité et de l'utilité de ces notions. Il aurait été agréable d'avoir aussi un aperçu des enseignements spécifiques aux formations technologiques STI et STL. De plus, cette séquence nous a permis, tout comme l'auto-évaluation guidée, de se rendre compte de la difficulté de l'évaluation/notation et de la difficulté de rendre compte d'un sujet en temps limité. Le projet professionel est aussi un élément attractif. Le travail effectué nous a généralement permis de confirmer notre projet professionnel. On regrettera tout de même le fort absentéisme à cette séquence, les absents étant plutôt ceux en difficulté, en ayant le plus besoin. »

Cet article rend compte de l'initialisation de notre démarche ; à suivre au prochain CETSIS ?

\section{Références bibliographiques}

[1] Démarche qualité : rapport de sélection au CETSIS 2001, Clermont-Ferrand, 29-30 octobre 2001.

[2] Cette remarque concerne les disciplines scientifiques, en vue de formations supérieures en sciences. 